\title{
STEP-BY-STEP DESIGN AND CONSTRUCTION OF VIRTUAL PLANNED ORTHOGNATHIC OCCLUSAL REPOSITIONING WAFER
}

\author{
Omar Hamad*, Anas Almukhtar**, Heba Abdalwahed Sleem ${ }^{* * *}$ and Amr Ghanem****
}

\begin{abstract}
Orthognathic surgery became a rapidly evolving procedure, innovations in virtual planning offers a potentially superior alternative to the conventional model surgery. The variation in techniques, virtual planning software's, imaging machines and 3D printing options made virtual planning an appealing field, however, the accuracy of these methods remains subject to accuracy assessment. A virtual planning technique was elaborated and validated. Both intermediate a final wafer of seven orthognathic patients were tested revealing mean deviation in intermediate wafer group of $0.64 \pm 0.33 \mathrm{~mm}$; while the mean deviation in final wafer group of $0.53 \pm 0.10 \mathrm{~mm}$
\end{abstract}

\section{INTRODUCTION}

Corrective jaw surgeries and orthognathic treatment are very useful in addressing both functional and aesthetic needs which are difficult or even impossible to treat with orthodontic procedures and conservative treatments ${ }^{(1)}$. Orthognathic surgery is defined as correction of deformities of the jaw and the associated malocclusion. Deformities include esthetic deformities, TMJ dysfunctions, deformities causing sleep apnea and others. These deformities are skeletal and extensive to be corrected or masked by orthodontic treatment alone.
For the past fifty years surgical techniques and planning methods have been evolving to treat developmental and acquired cranio-maxillofacial deformities restoring the symmetry, maxillo-mandibular relationships and facial dimensions ${ }^{(2)}$. Number of surgeons and practitioners using virtual reality and computer aided design and computer aided manufacture (CAD/CAM) to treat complex and challenging CMF case is increasing every day ${ }^{(3)}$. The improving quality of medical digital technology along with it becoming more feasible for most practitioner thus popularity reciprocally made it a rapidly improving field regarding the machinery and the software.

\footnotetext{
* Oral and Maxillofacial Department, Faculty of Dentistry, Ain shams university

** Assisstant Professor of Orthodontics

*** Professor of Oral and Maxillofacial Surgery Faculty of Dentistry Ain Shams University

**** Oral and Maxillofacial Department, Faculty of Dentistry, Ain Shams University
} 
The nature of orthognathic surgeries implies a very narrow window of errors that dictates an accurate delivery of the model plan to the operation room. Occlusal repositioning wafers or simply orthognathic wafers could be utilized to bridge the gap between them. The intermediate wafer allows the repositioning of the maxilla relative to the intact mandible (in case of maxilla first surgeries) and the final wafer aids in the achievement of the final planed position and occlusion. Wafers minimize or eliminate the intraoperative speculations and thus minimize the error ${ }^{(4)}$.

\section{METHODS}

A total of 7 patients underwent bimaxillary surgery. The mean age was 26.71 years (ranged between 20 and 31 years old). Two patients were males and 5 were females. All patients had preoperative orthodontic treatment.Amean of 3.14 2.96 mm maxillary advancement and $0.71 \pm 5.99 \mathrm{~mm}$ mandibular set back advancement was performed. Only 1 out of 7 patients was subjected to genioplasty. The data was collected from patients referred to the oral and maxillofacial department, faculty of dentistry, Ain Shams university for orthognathic evaluation and treatment. Ethics approval was obtained on the fourth of July 2017 from Faculty of Dentistry Ain Shams University Research Ethics Committee (FDASU-REC), code number (175).

A full skull cone beam CT of the patient was recorded using i-CAT (Imaging Sciences International Inc. Hatfield, PA) with the patient in natural head position and in rest position. These two parameters are critical to ensure a normal soft tissue contour without distortion.

Since most orthognathic patients are equipped with orthodontic brackets and wire an artifact is generated during imaging also enamel magnification is evident, that is why acquiring a teeth model free of artifact and magnification is mandatory. Impressions of both maxillae was recorded using alginate impression material (Tropicalgin, Zhermack, Italy) and casted with extra hard stone (Elite Master, Zhermack, Italy). Next step was to transfer this real cast to a virtual one. Two main techniques were available, laser scanning and CBCT. The later was the technique of choice for three mail reasons:

1. Consistence in imaging technique with minimum operator interference in contrast with intra oral laser scanner that needs manipulation to record all aspects of the casts.

2. Record of all aspects of the casts compared with scanner that will always has a limitation toward the surface hidden to the platform.

3. Availability.

Mimics (Materialise, Leuven, Belgium) and 3Matic (Materialise, Leuven, Belgium) were our software package of choice. Mimics software package offers a wide variety of functions and flexibility manipulating the models and it is considered as industrial gold standard by many authors ${ }^{(5)}$.

As shown in figure 1, DICOM files of the full skull record was imported into the software and thresholding performed to outline the hard tissues. A "mask" was ready to be converted into a 3D model of the skull. The 3D model was inspected to ensure that maximum amount of the skull bones was rendered with minimal artifact. Wrapping of the model can be performed to make the model solid and enhance its appearance. The rendered model had an obvious imperfection at the dental area. These were the inevitable streak artifacts caused by the magnification of the enamel and the artifact cause by the orthodontics braces. For an accurate planning and construction of surgical wafer, replacement of the dentition is a must, figure 2 .

The 3D model of the skull and that of the dental casts were available and ready. The 3D stereolithography (stl) model of the casts was imported into the software. A partial Procrustes point registration was performed to superimpose the dental models on the skull model. The skull model dentition was cropped leaving the dentition of the superimposed dental 
casts to take their place. The dentition of the casts is less magnified and artifact free. A hybrid model was established by the combination of the skull model and the dentition imported from the scanned plaster casts, figure 3 .

The alignment of the casts via point registration was further checked and inspected to ensure minimal inaccuracy. That was possible by inspecting the contour of the casts model in the axial, coronal and sagittal cuts.

Osteotomies do not have to replicate the exact path made in theatre although mimicking the real surgical osteotomies beforehand may highlight some issues that are beneficial during the surgery

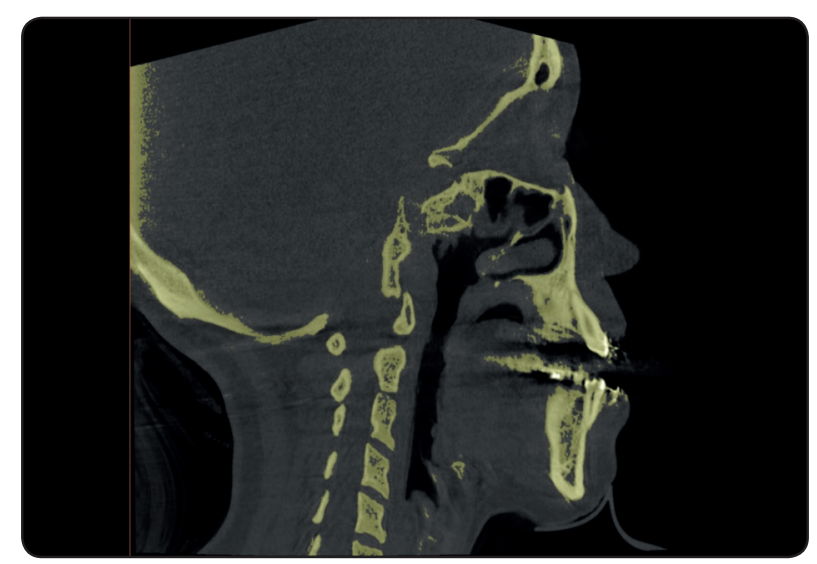

Fig. (1) Thresholding (selected Hounsfield unit is marked yellow)

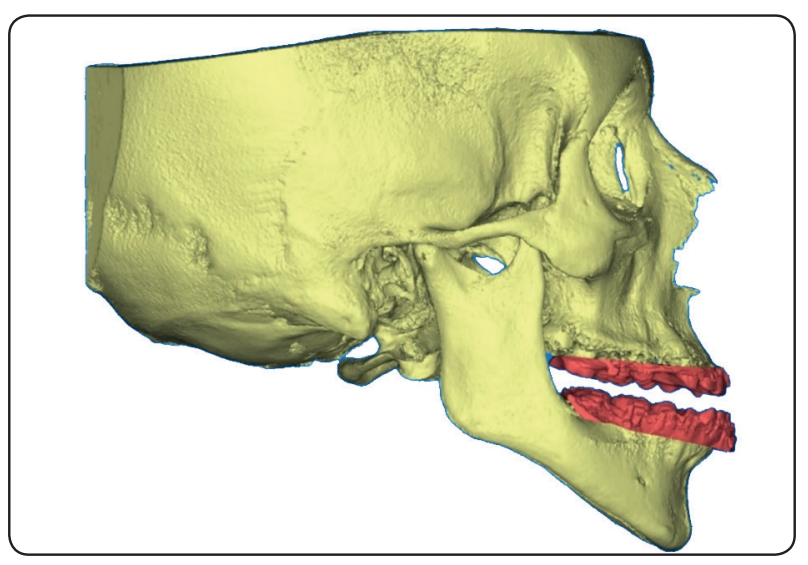

Fig. (3) Hybrid Model (replaced teeth are coded red) such as the proximity of the nerve bundle and the size of bony gaps and intersections. A virtual Le fort I osteotomy was made to separate the maxilla from the cranial base and a virtual BSSO was made to separate the body of the mandible from the rami, figure 4.

Segments were virtually displaced with the repositioning function, movements were measured in millimeters and rotations in degrees. The movements followed the surgical plan regarding which jaw was to be operated first. After moving the first jaw in position the intermediate wafer was fabricated. Then the opposing jaw was moved to the best occlusion and the final wafer was fabricated.

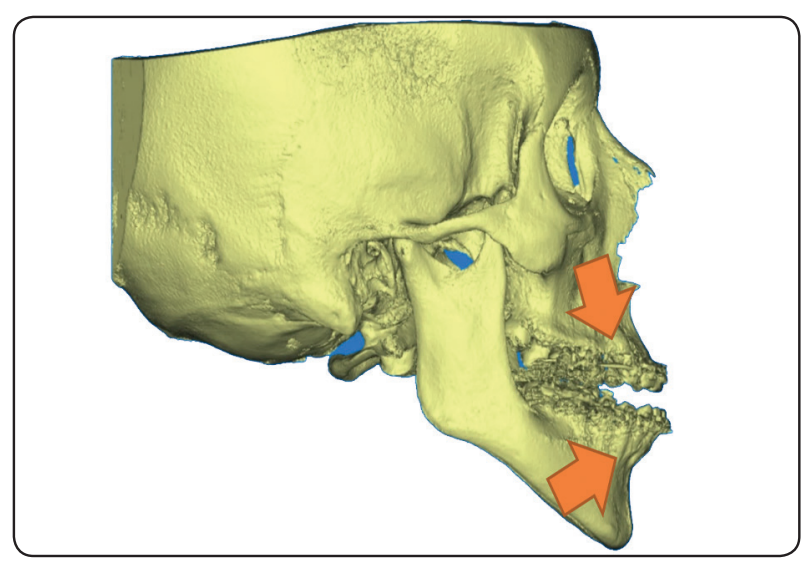

Fig. (2) 3D model of the bony skull (Artifact marked by arrows)

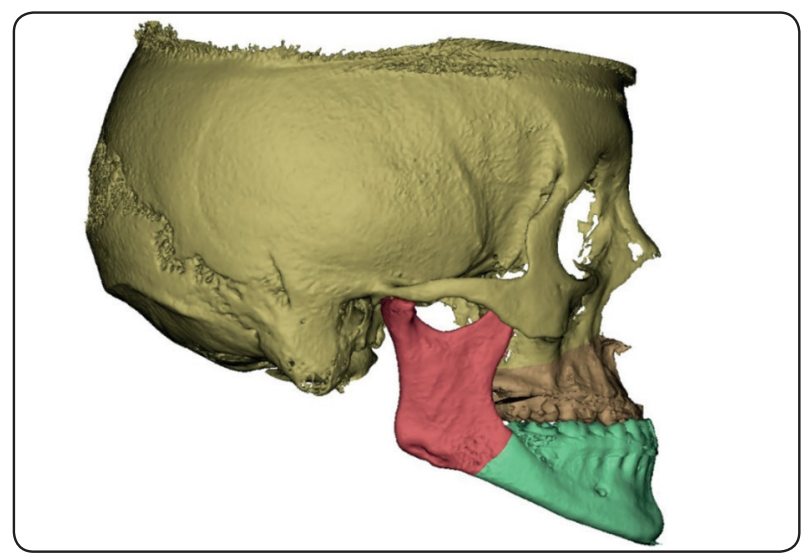

Fig. (4) Virtual osteotomies (different segments are color code for better visualization) 
Boolean function was used and the occlusion was subtracted out of the disc shaped object. By then, the disc had the negative impression of the occlusion and was trimmed just to the border of the orthodontic braces to ease the insertion in the mouth without interferences during the operation. Excessive materials in the buccal surface would be bulky and could disfigure the lips, on the other hand, a very thin wafer is liable to fracture during positing manipulation in theatre. The same procedures were applied to fabricate both the intermediate and the final wafers.

\section{Validation of the technique}

Quality of the occlusal fitting was inspected and evaluated. In case the wafer does not fit the teeth due to inaccurate design, distortion or shrinkage it would be useless during the operation. Wafers were checked on the dental casts and clinically on patient's teeth. The criteria for a successful wafer included the following:

1. Wafers are to fit the teeth passively without pressure.

2. No rocking should be detected.

3. No interference should be present.

4. Wafers fitting post operatively (only applicable for the virtual final wafer).

A novel method was designed and adopted to assess the accuracy of the wafers using a computer program. This method allowed to evaluate and compare the difference and degree of similarity between the conventional and virtual wafers without using the virtual wafers (tested wafers) to make actual changes to the patient.

Paired sample t-test was performed to compare accuracy between intermediate and final occlusal repositioning wafers within control and virtual technique groups. Chi-square test was conducted to compare frequency distribution of wafer checks outcomes between different wafer types and fabrication techniques.

\section{RESULTS}

The mean deviation representing error in intermediate wafer group was $0.64 \pm 0.33 \mathrm{~mm}$; while the mean deviation in final wafer group was $0.53 \pm 0.10 \mathrm{~mm}$. Paired sample t-test showed that there was no statistically significant difference in mean deviation between virtual and control group $(\mathrm{P}=0.403)$.

The average overall time needed for virtual planning of each case was 5.12 hours. This did not include time spent at the radiology department for imaging and $\mathrm{CBCT}$ acquisition.

Virtual wafers manufactured, only 1 out of 7 intermediate wafers showed incomplete seating and rocking, however all final wafers seated properly and revealed no rocking. One intermediate and one final wafer have shown some interferences.

Chi-square test revealed that there was no statistically significant difference in frequency distribution of wafer check points between intermediate and final wafers.

\section{DISCUSSION}

The conventional methods of planning maxillofacial and orthognathic surgeries have proven reliable for many years, however, new technological advances are shifting the field to a more effective and accurate approach that is yet to evolve. 3D visualization of anatomy as well as deformities and pathologies, saving of time, higher precision and better communication are among the many advantages of the virtual planning of orthognathic surgeries ${ }^{(6)}$.

The virtual model surgery technique was based on the methods elaborated by Shaheen et al. who used Pro Plan software (Materialise, Leuven, Belgium) ${ }^{(7)}$ which can be looked at as an orthognathic specific version of the Mimics (Materialise, Leuven, Belgium) that we used in our planning. The method described by Shaheen et al. for dental models 
registration via special scanning protocol as well as point registration reduced the scans the patient had to receive and preserved the soft tissue contour and the lip positions compared to other methods using multiple scan protocol and fiducial markers and bulky hardware ${ }^{(8-10)}$. The team also provided a solution that we adopted to avoid occlusal collision on the final splint by scanning the dental models in the final occlusion.

Bimaxillary patients were chosen for the research to allow assessment of both the intermediate and final wafers excluding patient suffering from systemic diseases and syndromes that would complicate the treatment planning and affect the results.

Adopting virtual planning and rapid prototyping was relatively new to our team. The literature is overwhelmed with different protocols of planning as well as software and techniques ${ }^{(11)}$, therefore validating the chosen technique before using it clinically and testing its accuracy in our hand was a must to avoid unnecessary complications to our patients. Studying seven patients seemed reasonable considering the relatively low rate of patient suffering bimaxillary deformities requiring orthognathic surgery and are motivated and willing to undergo the procedure, orthognathic surgery necessitate a certain degree of commitment and understanding of risk and benefits ${ }^{(12)}$.

In both the planning and assessment we chose the CBCT for the acquisition of the dental models and wafers, the accuracy of that modality was validated by Shaheen et al. who used a special cast protocol ${ }^{(8)}$, CBCT allowed imaging of the model from all sides with the same precision. It was feasible to separate the casts and the wafers on the assessment models using thresholding since the gypsum casts and printed wafer are different in density, a step that would be virtually impossible if a laser scanner or an optical scanner were used, the scanner would miss the record of the occlusal surface and would consider both material the same.
Elbokle and Sultan evaluated the virtually planned orthognathic wafers and compared them to conventional one. They used the Pro Plan software (Materialise, Leuven, Belgium) for the virtual plan. They measured the distance error of the printed virtual wafer after being superimposed on the virtual planned one in ten patients, $0.44 \mathrm{~mm}$ was the mean distance error measured ${ }^{(13)}$.

Shqaidef et al. tested the accuracy of their virtual technique on the data of ten patients and positioned a mandibular model on the maxilla using conventional wafer and stl printed wafer. He assigned landmarks and measure the distance which resents the error between both techniques, the absolute distance ranged from $0.04-1.73 \mathrm{~mm}^{(14)}$.

The virtually designed wafer tested by our team showed no statistically significant difference compared to the gold standard conventionally fabricated wafer for both intermediate and final wafers. The mean deviation in intermediate wafer group was $0.64 \pm 0.33 \mathrm{~mm}$; while the mean deviation in final wafer group was $0.53 \pm 0.10 \mathrm{~mm}$.

\section{CONCLUSION}

The virtual technique adopted by our team for construction of occlusal wafers showed good fitting and almost no rocking or interference, minor interferences were caused by the remnants of the supports made during printed to support the $3 \mathrm{D}$ model during the process, these interferences were easily eliminated in the occlusal trial session with a finishing stone. In further model that complication was reduced by orienting the models during printing and relocating printing supports away from the fitting surface of the wafers.

\section{REFERENCES}

1. Ayoub A. Handbook of orthognathic treatment: a team approach. Chichester, West Sussex, UK: John Wiley \& Sons, Ltd; 2014.

2. Bell RB. Computer Planning and Intraoperative Navigation in Orthognathic Surgery. J Oral Maxillofac Surg. 2011;69(3):592-605. 
3. Schramm A, Gellrich NC, Schmelzeisen R. Navigational surgery of the facial skeleton. Berlin ; New York: Springer; 2007. 170 p.

4. Bamber MA, Harris M. The role of the occlusal wafer in orthognathic surgery; a comparison of thick and thin intermediate osteotomy wafers. J Cranio-Maxillofac Surg. 1995; 23(6):396-400.

5. Yuan P, Mai H, Li J, Ho DC-Y, Lai Y, Liu S, et al. Design, development and clinical validation of computeraided surgical simulation system for streamlined orthognathic surgical planning. Int J Comput Assist Radiol Surg. 2017;12(12):2129-43.

6. Ayoub A, Khambay B. A paradigm shift in the diagnosis \& management of dentofacial deformities. Saudi Dent J. 2012;

7. Shaheen E, Coopman R, Jacobs R, Politis C. Optimized $3 \mathrm{D}$ virtually planned intermediate splints for bimaxillary orthognathic surgery: A clinical validation study in 20 patients. J Cranio-Maxillofac Surg. 2018;46(9):1441-7.

8. Shaheen E, Sun Y, Jacobs R, Politis C. Three-dimensional printed final occlusal splint for orthognathic surgery: design and validation. Int J Oral Maxillofac Surg. 2017;46(1):67-71.
9. Swennen GRJ, Mollemans W, De Clercq C, Abeloos J, Lamoral P, Lippens F, et al. A Cone-Beam Computed Tomography Triple Scan Procedure to Obtain a ThreeDimensional Augmented Virtual Skull Model Appropriate for Orthognathic Surgery Planning: J Craniofac Surg. 2009;20(2):297-307.

10. Gateno J, Xia J, Teichgraeber JF, Rosen A. A new technique for the creation of a computerized composite skull model. J Oral Maxillofac Surg. 2003;61(2):222-7.

11. Stokbro K, Aagaard E, Torkov P, Bell RB, Thygesen T. Virtual planning in orthognathic surgery. Int J Oral Maxillofac Surg. 2014;43(8):957-65.

12. AlKharafi L, AlHajery D, Andersson L. Orthognathic Surgery: Pretreatment Information and Patient Satisfaction. Med Princ Pract. 2014;23(3):218-24.

13. Elbokle N, Sultan O. The Precision of 3D Printed CAD/ CAM Occlusal Splints in Orthognathic Surgery. Egypt Dent J. 2018;64(3):2073-9.

14. Shqaidef A, Ayoub AF, Khambay BS. How accurate are rapid prototyped (RP) final orthognathic surgical wafers? A pilot study. Br J Oral Maxillofac Surg. 2014; 52(7):609-14. 